\title{
Quantifying and reporting cardiac findings in imaging of COVID-19 patients
}

\author{
Ajay Kumar Mishra', Amos Lal' ${ }^{2}$, Kamal Kant Sahu', Mark Kranis ${ }^{3}$, Jennifer Sargent ${ }^{1}$ \\ ${ }^{1}$ Department of Medicine, Saint Vincent Hospital, Worcester, MA; ${ }^{2}$ Department of Medicine, Division of Pulmonary \\ and Critical Care Medicine, Mayo Clinic, Rochester, MN; ${ }^{3}$ Department of Medicine, Division of Cardiovascular \\ Medicine, Saint Vincent Hospital, Worcester, MA, USA
}

\begin{abstract}
Severe acute respiratory syndrome coronavirus 2 (SARSCoV-2) infection continuous to be a public health emergency and a pandemic of international concern. As of April $31^{\text {st }}$, the reported cases of COVID-19 are three million in 186 countries. Reported case fatality has crossed 200 thousand among which more than fifty thousand has been in the USA. Most patients present with symptoms of fever, cough, and shortness of breath following exposure to other COVID-19 patients. Respiratory manifestations predominate in patients with mild, moderate, severe illness. Acute cardiac injury is the most commonly reported cardiac manifestation in patients with severe COVID19 and is diagnosed with the help of raised troponin. Imaging of patients with COVID-19 consistently reports various pulmonary parenchymal involvement. In this article we wanted to reinforce and review the various reported imaging patterns of cardiac and mediastinal involvement in COVID-19 patients. Among patients with COVID-9 who underwent various imaging of chest various cardiac findings including pericardial effusion,
\end{abstract}

Correspondence: Ajay Kumar Mishra, Department of Medicine, Saint Vincent Hospital, Worcester, MA, USA.

E-mail: Ajay.Mishra@stvincenthospital.com

Key words: COVID-19; cardiac; imaging.

Contributions: All the authors played a significant role in the paper. All authors have seen the manuscript and agree to the content and data.

Conflict of interest: The authors declare no conflict of interest.

Funding: None.

Received for publication: 19 May 2020.

Accepted for publication: 7 August 2020.

${ }^{\circ}$ Copyright: the Author(s), 2020

Licensee PAGEPress, Italy

Monaldi Archives for Chest Disease 2020; 90:1394

doi: 10.4081/monaldi.2020.1394

This article is distributed under the terms of the Creative Commons Attribution Noncommercial License (by-nc 4.0) which permits any noncommercial use, distribution, and reproduction in any medium, provided the original author(s) and source are credited. myocarditis, cardiomegaly has been reported. Most of these findings have been consistently reported in patients with significant acute myocardial injury, and fulminant myocarditis. Acute biventricular dysfunction has also been reported with subsequent improvement of the same following clinical improvement. Details of cardiac MRI are rather limited. In a patient with clinical presentation of acute myocarditis, biventricular myocardial interstitial edema, diffuse biventricular hypokinesia, increased ventricular wall thickness, and severe LV dysfunction has been reported. Among patients with significant clinical improvement in LV structure and function has also been documented. Multiple cardiovascular comorbidities, clinical features, laboratory parameters, and imaging findings are shown to contribute to poor outcome in COVID-19 patients. With increasing number of clinical cases, future imaging studies will be instrumental in identifying the various cardiac manifestations, and their relation to clinical outcome.

\section{Introduction}

Severe acute respiratory syndrome coronavirus 2 (SARSCoV-2) infection continues to be a public health emergency and a pandemic of international concern. As of April 31 ${ }^{\text {st }}$, the reported cases of COVID-19 are three million in 186 countries. Reported case fatality has crossed 200 thousand among which more than fifty thousand has been in the USA. Most patients present with symptoms of fever, cough, and shortness of breath following exposure to other COVID-19 patients [1]. Respiratory manifestations predominate in patients with mild, moderate, severe illness. Imaging of patients with COVID-19 consistently report various pulmonary parenchymal involvement [2]. Available data on chest imaging, focusing on pulmonary manifestations is extensive [3]. In the recent review by Cao et al. the authors have reviewed 31 articles and have discussed the various clinical, radiological and outcome details of various pulmonary findings in 46959 patients with computed tomography chest from literature [4]. In another study by Zhu et al., the authors reviewed 34 retrospective studies and reported CT imaging features of 4121 COVID-19 patients [5]. The authors identified that the chest CT findings of bilateral, multilobar, patchy and groundglass opacities were most common uniformly across all the studies [3-5]. Most authors reporting chest CT findings have done an excellent description of pulmonary manifestation of COVID-19 patients [4-7]. In this article we wanted to reinforce and review the various reported imaging patterns of cardiac and mediastinal involvement in COVID-19 patients. 


\section{Methodology}

In this review, we included articles on COVID-19 as published in Medline. We used the terms "COVID-19" and "cardiovascular diseases" under the Mesh database in PubMed. To be included in our review articles had to have details of cardiac imaging in patients with COVID-19. Imaging modalities that were included are chest X-ray, computed tomography (CT) thorax, magnetic resonance imaging (MRI), echocardiography, nuclear imaging. Case reports, case series, retrospective, and prospective observational studies on COVID-19 patients were eligible to be included. Studies lacking patient details, cardiac imaging findings were excluded. We also excluded opinions, management guidelines, reviews, recommendations that did not include patients with cardiac imaging detail. Two independent clinicians were involved with the screening of the articles.

\section{Results}

We identified 59 articles on "cardiovascular" and "COVID19 " as published in Pub Med by $31^{\text {st }}$ April 2020. Among these only 2 studies described details of echocardiographic findings in the patients. One case series reported details of coronary angiogram. We did not find any other study of COVID-19 patients reporting cardiac imaging. We included details from other case reports, case series, and letters that reported any detail of cardiac imaging. We have described the details below.

\section{Discussion}

\section{Cardiac manifestations}

Most studies have reported new onset cardiovascular involvement in around 10-12.5\% of patients [8,9]. Details of electrocardiography (EKG) and echocardiography (ECHO) is limited and is only reported in a subset of patients. Among patients with COVID19 who underwent various imaging of chest various cardiac findings including pericardial effusion, myocarditis, cardiomegaly has been reported $[4,5,8]$.

\section{Pathophysiology of cardiac injury in COVID-19}

In patients with COVID-19, multiple mechanisms of myocardial injury have been postulated. Cardiac injury can be subdivided into ischemic, and nonischemic etiology [10].

\section{Nonischemic injury}

Nonischemic injury has been reported extensively in the literature. The following mechanisms of nonischemic myocardial injury have been reported in patients with COVID-19: i) Viral myocarditis: SARS -CoV-2 enters inside the target cells using membrane-bound angiotensin-converting enzyme two (ACE-2) receptors. Predominant pulmonary manifestations are secondary to the expressed level of ACE-2 in type I and type II pulmonary alveolar epithelium [11]. ACE-2 is also expressed in the cardiac myocytes, cardiac endothelium, and vascular smooth muscle cells. Even though viral attachment to these receptors has been considered, the exact mechanism by which this initiates and precipitates myocardial injury is unknown [12]. However, autopsy studies have shown features of cardiac myocyte injury, lymphocytic myocarditis, and pericarditis [13]. There have been reports of progression of myocardial involvement to fulminant myocarditis as well. ii) Cytokine storm: cytokine storm has been defined as dysfunctional, uncontrolled, exaggerated activation of the immune system, resulting in excessive cytokine release, following COVID-19 infection [14]. In patients with severe COVID-19 disease, cytokine storm has been diagnosed by increased levels of inflammatory markers in plasma. Various inflammatory markers including C-reactive protein (CRP), erythrocyte sedimentation rate (ESR), ferritin, procalcitonin, D- dimer, interleukins (IL-2, IL-7), IgG induced protein 10, granulocytecolony-stimulating factor, tumor necrosis alpha and chemokine ligand 3 have been reported to be elevated [15]. iii) Prothrombotic state: Resulting in elevated d-dimer, fibrinogen, lactate dehydrogenase, and other coagulation markers. This is further accentuated with the presence of thrombocytopenia, disseminated intravascular coagulation, and deranged coagulation parameters [16,17].iv) Other postulated mechanisms have been: a) hypoxia-induced cardiac myocyte injury and apoptosis, b) viral infection precipitating secondary hemophagocytic lymphohistiocytosis, c) stress-induced cardiomyopathy, d) underlying electrolytes, metabolic imbalances, e) injury secondary to adverse drug reactions $[15,18,19]$.

\section{Ischemic injury}

The ischemic mechanism of myocardial injury has been reported rarely. The occurrence of both type I and type II myocardial infarction has been postulated. Infection is thought to precipitate plaque disruption directly and result in myocardial infarction (type I) in the background of inflammation-induced upregulation of prothrombotic and procoagulant state. Myocardial oxygen supplydemand mismatch can result in myocardial infarction (type II). Precipitators of this mismatch are thought to be cytokine storm, hypoxia, acidosis, sepsis, shock, and abnormal blood pressure [20].

\section{Clinical presentation}

Patients with severe COVID-19, can present with several cardiac manifestations. Acute cardiac injury as defined by the raised cardiac enzymes, with and without EKG changes contributes to the diagnosis [21]. Figure 1 depicts the various cardiac findings that have been reported in patients with COVID-19. Patients presenting with cardiovascular involvement tend to present with shortness of breath, fatigue, palpitations, chest pain along with the constitutional symptoms [22]. Diagnosis of cardiac involvement is established with raised levels of highly sensitive cardiac troponin, creatine kinase MB, creatinine phosphokinase, NT proBNP, lactate dehydrogenase, and d-dimer. Various imaging modalities as discussed below contribute to diagnostic evaluation, management, prognosis [19-21].

\section{Chest X-ray}

Significant cardiac findings in chest X-ray (CXR) has been limited. Enlargement of cardiac silhouette has been reported in 2 distinct reports. Dabbagh et al. have reported a 67 years-old lady presenting with COVID-19 and evidence of cardiomegaly in CXR [23]. Similarly in another report 37 years-old gentleman with COVID-19 had cardiomegaly in CXR [24]. While pericardial tamponade was evident in the first report, the latter was diagnosed 
with fulminant myocarditis. Both these patients had significant clinical improvement, and normalization of cardiac silhouette was documented in the patient with fulminant myocarditis [23,24].

\section{Echocardiography}

Reports of patient with ECHO findings continue to increase $[25,26]$. A recent study by Deng et al. reported ECHO findings of 112 patients with COVID-19 [25]. Sixty-seven of these patients was reported to have severe illness. In the study standardized ECHO parameters were reported. An abnormal ECHO was defined by: i) left ventricular ejection fraction (LVEF) less than $50 \%$, ii) Presence of segmental wall motion abnormalities, iii) Left ventricular wall thickness of more than $10 \mathrm{~mm}$, iv) Presence of pericardial effusion of more than equal to $5 \mathrm{~mm}$. Peak tricuspid regurgitation velocity of more than $2.8 \mathrm{~m} / \mathrm{s}$ was used to define pulmonary hypertension. Using these parameters they reported $8.9 \%(n=10)$ of patient's with evidence of cardiac abnormality [25]. Presence of pericardial effusion, pulmonary hypertension, reduced LVEF, an evidence of segmental wall abnormality, and increased LV wall thickness was identified in $19.6 \%(n=22), 13.4 \%(n=15), 5.4 \%$ $(n=6), 4.5 \%(n=5)$, and $2.7 \%(n=3)$ patients. The authors also reported that pericardial effusion, pulmonary artery hypertension, reduced ejection fraction, and segmental wall motion abnormality was higher in patients with severe COVID-19 illness [25]. In the study composite endpoint of outcome included: i) an admission to intensive care unit, or ii) requirement of mechanical ventilation, or iii) requirement of extracorporeal membrane oxygenation, or iv) death. Interestingly the authors noted that each of these ECHO findings were statistically significant $(\mathrm{p}<0.01)$ in contributing to the composite endpoint of the study. However, the authors failed to report how many of these patients had a pre-existing cardiac abnormality. They also did not report the differences in outcome among patients with and without preexisting cardiac abnormality [25].

Another study reported that presence of right ventricular longitudinal strain pattern in ECHO was independently related to higher mortality. Other commonly reported ECHO findings have been diffuse myocardial wall motion abnormality, left ventricular enlargement, Takotsubo cardiomyopathy, signs of increase in right atrial pressure including increased inferior vena cava (IVC) diameter and decreased IVC collapsibility $[25,26]$. Most of these findings have been consistently reported in patients with significant acute myocardial injury, and fulminant myocarditis. Among patients with significant clinical improvement in LV structure and function has also been documented [26-29].

\section{Computed tomography chest}

Cardiac involvement is being frequently reported in computed tomography (CT) chest. In a study including 83 patients who underwent CT chest, pericardial effusion was reported in $4.8 \%$ of patients $[7,9,30]$. Pericardial effusion in the above study was mild and did not progress. Among patients who had clinical improvement, there was documentation of improvement of pericardial effusion [7]. Multiple other studies have also reported the presence of pericardial effusion in COVID-19 patients. Interestingly in the first study the presence of pericardial effusion was noted to correlate well with the severity of the disease [9]. Evidence of myocarditis including increased wall thickness, myocardial edema and hypokinesia has been reported in various reports of COVID-
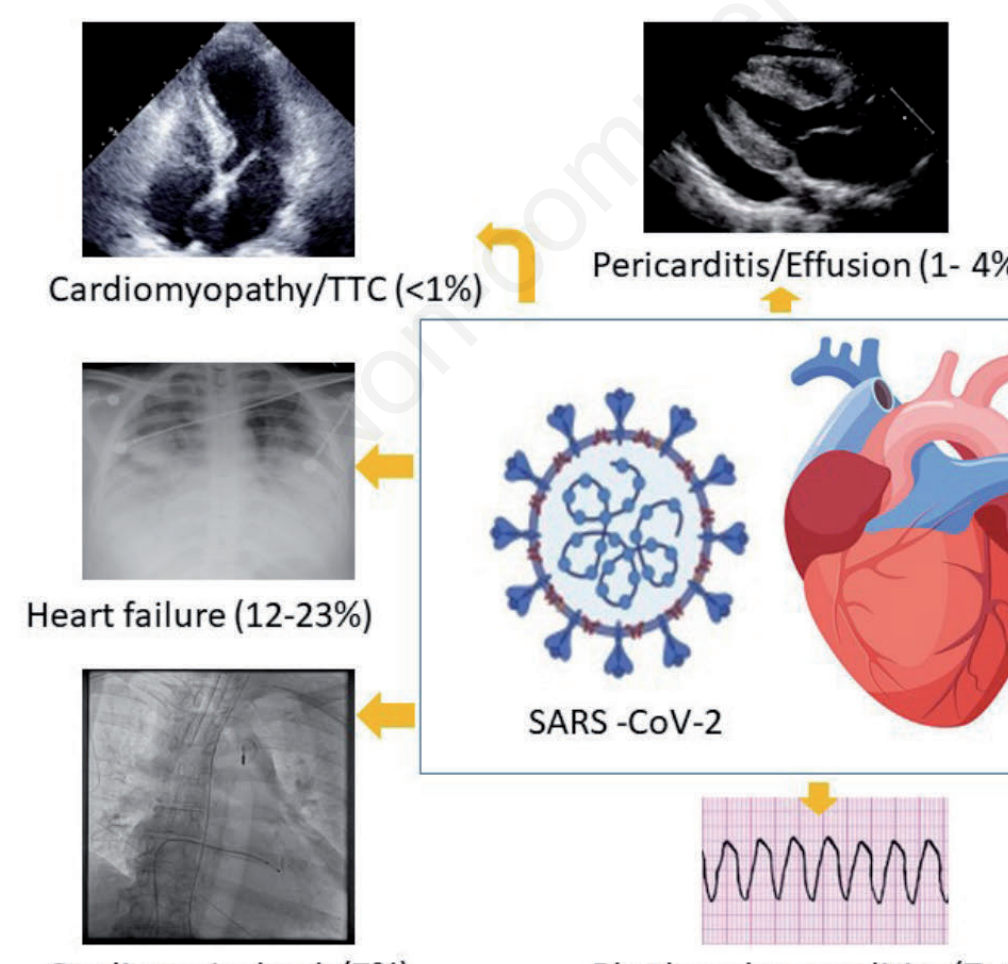

Cardiogenic shock (7\%)

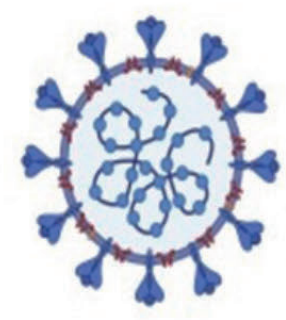

SARS -CoV-2
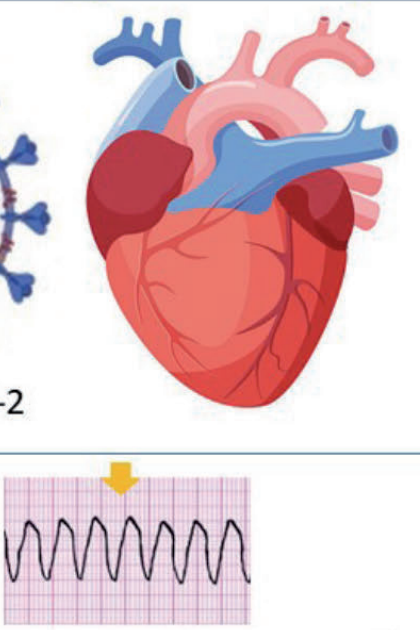

Rhythm abnormalities (7 -16 \%)

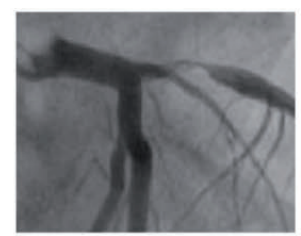

Acute Coronary Syndromes ( $<1 \%)$

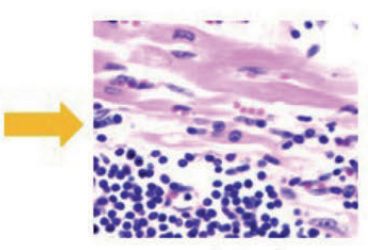

Myocarditis (8-12\%)

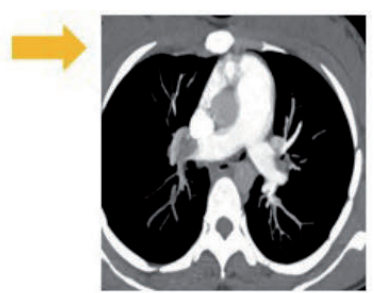

Thromboembolic event (22\%)

TTC: Takotsubo cardiomyopathy

Figure 1. The incidence and patterns of myocardial alteration in patients with COVID-19. 
19 patients. Acute biventricular dysfunction has also been reported with subsequent improvement of the same following clinical improvement $[7,9,30]$.

\section{Coronary angiography}

In the recent study including 18 patients of COVID-19 with ST segment elevation on EKG, from 6 New York hospitals, authors have reported detailed reports of coronary angiography (CAG) in $50 \%$ $(n=9)$ patients [31]. Sixty-seven percent $(n=6)$ of the patient were identified to have obstructive coronary artery disease and $57 \%$ of the patient underwent percutaneous coronary intervention. Obstruction of both the coronary artery were reported. In this study mortality was higher among patients with normal CAG suggesting noncoronary myocardial injury [31]. While left ventriculogram has revealed basal hyperkinesis and apical ballooning suggesting Takotsubo cardiomyopathy in an 85-year-old lady with COVID-19 anxiety, details of left ventriculogram is limited in other studies $[32,33]$.

\section{PET CT}

Evidence of cardiac involvement has not been reported in a study including 4 patients, who underwent 18 F-FDG PET study [34].

\section{Cardiac MRI}

Details of cardiac MRI are rather limited. In a patient with clinical presentation of acute myocarditis, biventricular myocardial interstitial edema, diffuse biventricular hypokinesia, increased ventricular wall thickness, and severe LV dysfunction has been reported [9].

\section{Mediastinal involvement}

Consistently studies have reported significant mediastinal lymphadenopathy in patients with COVID-19. Presence of lymphadenopathy has been reported in $5.4 \%$ of patients [5]. Mediastinal involvement is not reported in CXR in view of limited visibility and extensive pulmonary parenchymal involvement in patients with severe illness. In a recent meta-analysis including 34 retrospective studies on chest CT, lymphadenopathy was noted to be reported in 8 retrospective studies [5]. In one report of COVID-19 patients who underwent PET CT sites of lymphadenopathy that have been reported are mediastinal, hilar and supraclavicular regions [34,35]. However, size, number and a specific subgroup of lymph node involvement have not been reported to predict disease severity or outcome.

\section{Cardiovascular involvement and outcome}

Reported mortality among patients with COVID-19 continues to change. The number of cases continues to rise in the US and, at the date we have conducted our study, the reported mortality has been $3.9 \%$. Patients with cardiovascular involvement are reported to have a poor outcome [8]. Poor outcome is defined as the development of ARDS, acute kidney injury, coagulopathy, worsening radiological changes, the requirement of intensive care unit admission, noninvasive, invasive ventilation, and mortality [36,37]. Predictors of worst outcome in patients with cardiac involvement

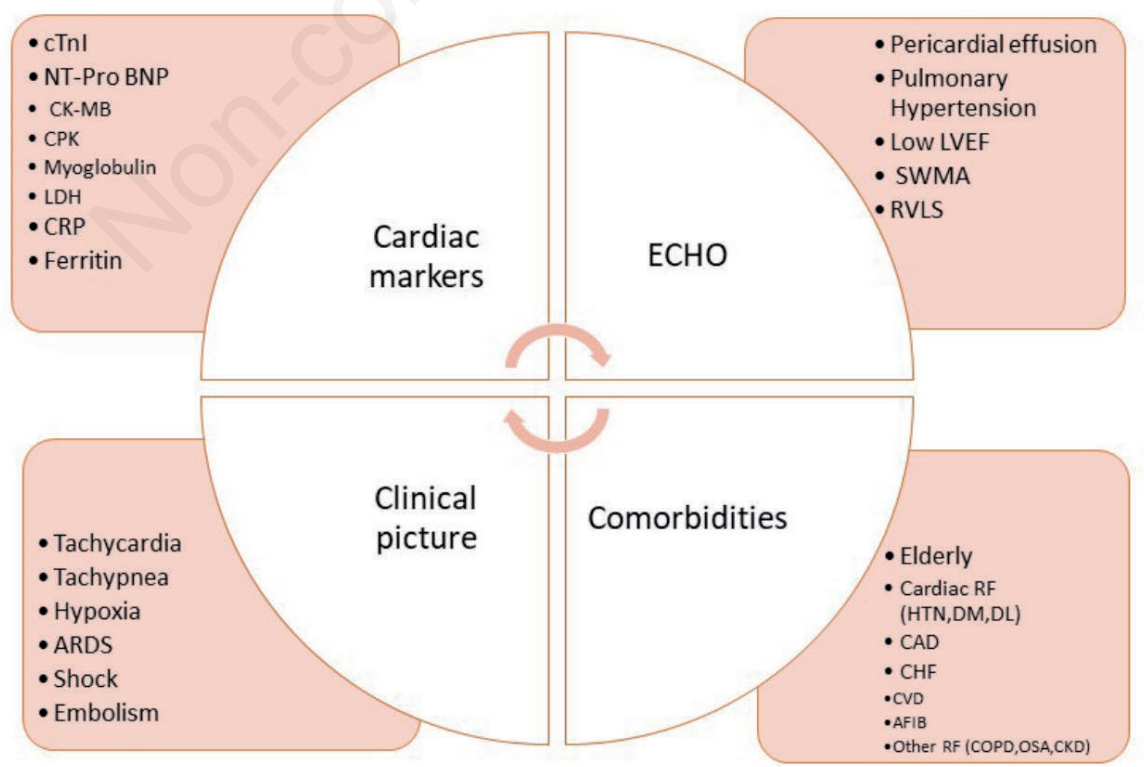

CTnl: Cardiac Troponinl, NT-Pro ENP:N terminal Pro Brain Natriuretic Peptide, CK-MB: Creatine Kinase MB, CPK: Creatine Phosphokinase, LDH: Lactate Dehydrogenase, CRP:C Reactive Protein, ARDS: Acute Respiratory Distress Syndrome, LVEF: Left Ventricular Ejection Fraction, SWMA: Segmental wall motion abrormality, RVIS: Right Ventricular Longitudinal Strain, RF: Risk Factors, HTN: Hypertension, DM: Diabetes Meli litus, DL: Dysipidemia, CAD: Coronary Artery Disease, CHF: Chroric HeartFailure, CVD. Cerebrovascular disease, AFIB: Atrialffibrillation, SWMA: Segmental wall motion abnormalty, RVLS: Right Ventricular Longitudinal Strain, RF: Risk Factors, HNN: Hypertension, DM: Diabe,
COPD: Chronic Obstructive Pulmonary Disease, OSA: Obstructive SleepApnea, CKD: Chronic Kidney Disease, ECHO: Echocardiography

Figure 2. Cardiovascular factors contributing to poor outcome in patients with COVID-19. 
can be sub-grouped into comorbidities, clinical features, laboratory parameters, and imaging findings as shown in Figure 2.

\section{Future prospective}

With patients' number reaching nearly 3 million in 186 countries, and reported mortality of around $6 \%$, most studies to date are consistently reporting pulmonary findings in chest imaging [38]. While it is clear that the presence of cardiovascular comorbidities like hypertension, heart failure, past history of coronary artery disease, cerebrovascular involvement, and acute cardiac injury is an independent predictor of poor outcome and mortality, it is surprising that most imaging studies report limited cardiac findings [19,20,25]. Similarly, lymphopenia, and leukocytosis are also a surrogate marker for severe illness in the background of a cytokine storm. However, few studies report the pattern of lymphadenopathy in COIVD-19 patients [34,35]. It is prudent to have details of various cardiac parameters including structure (chamber diameter, wall thickness, valves, supporting structures), functions, pericardial involvement, pulmonary artery diameter, IVC, major vessels, and details of various lymph node groups' involvement in patients who underwent various forms of imaging of chest for COVID-19. The cardiovascular implications of various medications that are used in treatment of COVID-19 can also be demonstrated with these imaging. With increasing number of clinical cases, future imaging studies will be instrumental in identifying the various cardiac manifestations, and their relation to clinical outcome [39-42].

\section{References}

1. Sahu KK, Mishra AK, Lal A. COVID-2019: update on epidemiology, disease spread and management. Monaldi Arch Chest Dis 2020;90:1292. doi: 10.4081/monaldi.2020.1292.

2. Sahu KK, Mishra AK, Lal A. Novel coronavirus (2019-nCoV): Update on 3rd coronavirus outbreak of 21 st century. QJM Mon J Assoc Physicians 2020;113:384-6.

3. Lal A, Mishra AK, Sahu KK. CT chest findings in coronavirus disease-19 (COVID-19). J Formos Med Assoc 2020;119:1000-1.

4. Cao Y, Liu X, Xiong L, Cai K. Imaging and clinical features of patients with 2019 novel coronavirus SARS-CoV-2: A systematic review and meta-analysis. J Med Virol 2020;92:1449-59.

5. Zhu J, Zhong Z, Li H, et al. CT imaging features of 4121 patients with COVID-19: A meta-analysis. J Med Virol 2020;92:891-902. doi: 10.1002/jmv.25910.

6. Sahu KK, Lal A, Mishra AK. An update on CT chest findings in coronavirus disease-19 (COVID-19). Heart Lung 2020;49:442-3. doi: 10.1016/j.hrtlng.2020.03.007.

7. Li K, Wu J, Wu F, et al. The clinical and chest CT features associated with severe and critical COVID-19 pneumonia. Invest Radiol 2020;55:327-31.

8. Mishra AK, Sahu KK, Lal A, Sargent J. Patterns of heart injury in COVID - 19 and relation to outcome. J Med Virol 2020. doi: 10.1002/jmv. 25847

9. Inciardi RM, Lupi L, Zaccone G, et al. Cardiac involvement in a patient with Coronavirus disease 2019 (COVID-19). JAMA Cardiol 2020;5:819-24.

10. Mishra AK, Sahu KK, Lal A, Sargent J. Mechanisms of stroke and the role of anticoagulants in COVID-19. J Formos Med Assoc 2020. doi: 10.1016/j.jfma.2020.06.026
11. Sahu KK, Mishra AK, Martin K, Chastain I. COVID-19 and restrictive lung disease: A deadly combo to trip off the fine balance. Monaldi Arch Chest Dis 2020;90:1346. doi: 10.4081/monaldi.2020.1346.

12. Mishra AK, Sahu KK, George AA, et al. Cerebrovascular events in COVID-19 patients. Monaldi Arch Chest Dis 2020;90:1341. doi: 10.4081/monaldi.2020.1341.

13. Buja LM, Wolf DA, Zhao B, et al. The emerging spectrum of cardiopulmonary pathology of the coronavirus disease 2019 (COVID-19): Report of 3 autopsies from Houston, Texas, and review of autopsy findings from other United States cities. Cardiovasc Pathol 2020;48:107233.

14. Sahu KK, Mishra AK, Martin K, Chastain I. COVID-19 and clinical mimics. Correct diagnosis is the key to appropriate therapy. Monaldi Arch Chest Dis 2020;90:1327. doi: 10.4081/monaldi.2020.1327

15. Mishra AK, Sahu KK, Lal A. Reporting of all cardiac medications and their outcome in COVID-19. J Med Virol 2020. doi: 10.1002/jmv. 25843

16. Klok FA, Kruip MJHA, van der Meer NJM, et al. Incidence of thrombotic complications in critically ill ICU patients with COVID-19. Thromb Res 2020;191:145-7.

17. Sahu KK, Mishra AK, Lal A. A twin challenge to handle: COVID-19 with pregnancy. J Med Virol 2020. doi: 10.1002/ jmv. 25784

18. Mishra AK, George AA, Sargent J. Letter to the Editor regarding "Acute Stroke management during the Coronavirus disease 2019 (COVID-19) pandemic: From trough of disillusionment to slope of enlightenment". World Neurosurg 2020;142:551-2. doi: 10.1016/j.wneu.2020.06.111

19. Kang Y, Chen T, Mui D, et al Cardiovascular manifestations and treatment considerations in COVID-19. Heart 2020;106:1132-41.

20. Sandoval Y, Januzzi Jr JL, Jaffe AS. Cardiac troponin for the diagnosis and risk-stratification of myocardial injury in COVID-19: JACC Review Topic of the Week. J Am Coll Cardiol 2020;76:1244-58.

21. Mishra AK, Lal A, Sahu KK, et al. Letter to the Editor regarding "Neurological Impact of Coronavirus Disease (COVID19): Practical considerations for the neuroscience community". World Neurosurg 2020;142:533-4. doi: 10.1016/j.wneu. 2020.05.089

22. Sahu KK, Mishra AK, Lal A. Trajectory of the COVID-19 pandemic: chasing a moving target. Ann Transl Med 2020;8:694. doi:10.21037/atm-20-2793.

23. Dabbagh MF, Aurora L, D'Souza P, et al. Cardiac tamponade secondary to COVID-19. JACC Case Rep 2020;2:1326-30. doi: 10.1016/j.jaccas.2020.04.009

24. Hu H, Ma F, Wei X, Fang Y. Coronavirus fulminant myocarditis saved with glucocorticoid and human immunoglobulin. Eur Heart J 2020. doi: 10.1093/eurheartj/ehaa190

25. Deng Q, Hu B, Zhang Y, et al. Suspected myocardial injury in patients with COVID-19: Evidence from front-line clinical observation in Wuhan, China. Int J Cardiol 2020;311: 116-21.

26. Li Y, Li H, Zhu S, et al. Prognostic value of right ventricular longitudinal strain in patients with COVID-19. JACC Cardiovasc Imaging 2020. doi: 10.1016/j.jcmg.2020. 04.014

27. Mishra AK, Mani S, George AA, Sudarsanam TD. Recurrent pericardial effusion and tamponade in a patient with ErdheimChester disease (ECD). BMJ Case Rep 2015;2015:bcr 2015212483. 
28. Shi S, Qin M, Shen B, et al. Association of cardiac injury with mortality in hospitalized patients with COVID-19 in Wuhan, China. JAMA Cardiol 2020;5:802-10.

29. Driggin E, Madhavan MV, Bikdeli B, et al. Cardiovascular considerations for patients, health care workers, and health systems during the Coronavirus disease 2019 (COVID-19) Pandemic. J Am Coll Cardiol 2020;75:2352-71.

30. Ye Z, Zhang Y, Wang Y, et al. Chest CT manifestations of new coronavirus disease 2019 (COVID-19): a pictorial review. Eur Radiol 2020;30:4381-9.

31. Bangalore S, Sharma A, Slotwiner A et al. ST-segment elevation in patients with Covid-19 - a case series. N Engl J Med 2020;382:2478-80.

32. Mishra AK, Sahu KK, Lal A, Peng Z. Wellens syndrome: Differential and outcome, QJM 2019;112:825-6.

33. Chadha S. 'COVID-19 pandemic' anxiety induced Tako-tsubo cardiomyopathy. QJM 2020;113:488-90.

34. Qin C, Liu F, Yen T-C, Lan X. 18F-FDG PET/CT findings of COVID-19: a series of four highly suspected cases. Eur J Nucl Med Mol Imaging 2020;47:1281-6.

35. Li K, Wu J, Wu F, et al. The clinical and chest CT features associated with severe and critical COVID-19 pneumonia. Invest Radiol 2020;55:327-31.

36. Guzik TJ, Mohiddin SA, Dimarco A, et al. COVID-19 and the cardiovascular system: implications for risk assessment, diagnosis, and treatment options. Cardiovasc Res 2020. doi:10.1093/cvr/cvaa106

37. Mishra AK, Lal A, Sahu KK, Sargent J. Cardiovascular factors predicting poor outcome in COVID-19 patients. Cardiovasc Pathol 2020;49:107246.

38. Sahu KK, Lal A, Mishra AK. Latest updates on COVID-2019: A changing paradigm shift. J Med Virol 2020;92:533-5.

39. Mishra AK, Sahu KK, Sargent J. Cardiac drugs and outcome in COVID-19. QJM 2020;113:523-4.

40. Mishra AK, Sahu KK, Lal A. Reporting of all cardiac medications and their outcome in COVID-19. J Med Virol 2020. doi: 10.1002/jmv.25843.

41. Zheng YY, Ma YT, Zhang JY, Xie X. COVID-19 and the cardiovascular system. Nat Rev Cardiol 2020;17:259-260.

42. Mishra AK, Sahu KK, Lal A, et al. A review of cardiac manifestations and predictors of outcome in patients with COVID19. Heart Lung 2020. doi: 10.1016/j.hrtlng.2020.04.019 